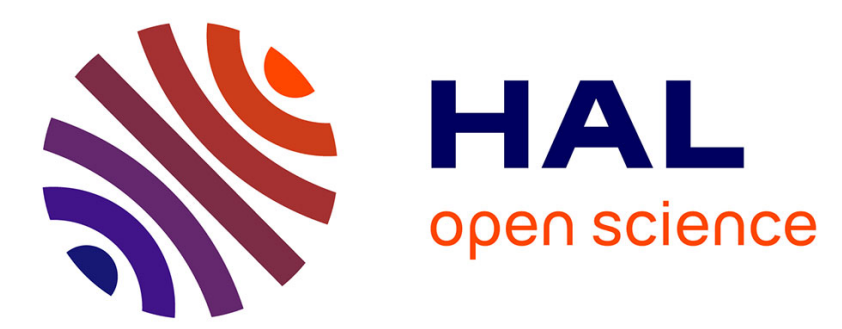

\title{
Heat transport and the Boltzmann equation in the theory of thermal boundary resistance \\ N. Perrin
}

\section{To cite this version:}

N. Perrin. Heat transport and the Boltzmann equation in the theory of thermal boundary resistance. Journal de Physique Lettres, 1977, 38 (7), pp.167-170. 10.1051/jphyslet:01977003807016700 . jpa00231351

\section{HAL Id: jpa-00231351 https://hal.science/jpa-00231351}

Submitted on 1 Jan 1977

HAL is a multi-disciplinary open access archive for the deposit and dissemination of scientific research documents, whether they are published or not. The documents may come from teaching and research institutions in France or abroad, or from public or private research centers.
L'archive ouverte pluridisciplinaire HAL, est destinée au dépôt et à la diffusion de documents scientifiques de niveau recherche, publiés ou non, émanant des établissements d'enseignement et de recherche français ou étrangers, des laboratoires publics ou privés. 


\title{
HEAT TRANSPORT AND THE BOLTZMANN EQUATION IN THE THEORY OF THERMAL BOUNDARY RESISTANCE
}

\author{
N. PERRIN
}

Groupe de Physique des Solides (*), Ecole Normale Supérieure, 24 rue Lhomond, 75231 Paris 05, France

(Reçu le 19 janvier 1977, accepté le 8 mars 1977)

\begin{abstract}
Résumé. - Nous considérons un flux de chaleur stationnaire à travers une plaque métallique en contact avec l'hélium liquide, et étudions les variations spatiales des flux de chaleur des phonons et des électrons au voisinage de l'interface solide-hélium, en résolvant simultanément l'équation de Boltzmann des phonons et l'équation de bilan d'énergie des électrons. Une température moyenne de phonons, définie dans chaque mode, présente un comportement non linéaire. Nous discutons l'incidence de ce comportement sur la résistance de Kapitza.
\end{abstract}

Abstract. - We consider a steady state heat flux flowing through a metallic slab in contact with liquid helium, and study the spatial variations of the phonon and electron heat fluxes in the vicinity of the solid-helium interface, by solving simultaneously the phonon Boltzmann equation and the electron energy balance equation. A mean phonon temperature, defined in each mode, presents a non linear behaviour. The influence of the latter on the Kapitza resistance is discussed.

1. Introduction. - The experimentally observed Kapitza resistance $R_{\mathrm{K}}$ between a solid and liquid helium is still a puzzling problem [1,2]. The first theory due to Khalatnikov [3], and based on the idea that thermal exchange is by phonons, failed to provide even the right order of magnitude of $R_{\mathrm{K}}[1,2,4]$. This theory proposes an acoustic mismatch model for the phonons [2], and assumes thermal equilibrium on each side of the interface. The uniform, but different temperatures of helium and solid, and the resulting temperature drop at the interface produces the thermal boundary resistance. Several theoretical attempts to provide mechanisms yielding a smaller $R_{\mathrm{K}}$ than that predicted by Khalatnikov have been made [5]. Challis et al. [6] considered the acoustic matching provided by the formation of a dense layer of helium at the interface. The resulting enhancement of the phonon transmission is however not sufficient to resolve the discrepancy. Other authors $[7,8]$ have investigated theoretically the effect of phonon attenuation in the solid, which leads to an opening of the critical cone predicted by the acoustic theory. However, the developed theory does not seem to be valid for temperatures $\gtrsim 1 \mathrm{~K}$ [8]. A combination of the two latter mechanisms has also been considered by Opsal and Pollack [9]. More recently, Cheeke and Ettinger [10] have considered the Van der Waals

(*) Laboratoire associé au Centre National de la Recherche Scientifique. layer as an acoustically absorbing medium. Whereas agreement with experiment is actually observed for some materials (copper) in the temperature range $0.1 \mathrm{~K}-1 \mathrm{~K}$, significant disagreement occurs for others (silicon).

In metals, the question arises of the direct or indirect contribution of electrons. Theoretical [11-13] as well as experimental information $[14,15]$ has been reported. Direct electron-helium processes involving the coupling between the surface modes and the conduction electrons in the metal have been considered [11, 12], and some experiments interpreted as evidence of these electronic processes [14]. More recently, it was suggested [13] that the magnetic field dependence of the thermal conductance related in the works of Wagner et al. [14] and Challis et al. [15] will exist even if the thermal boundary conductance is dominated by acoustic processes.

In this letter, we use an approach to the Kapitza problem based on transport technique and therefore different from the above acoustic theories relying on the thermal equilibrium properties of each material in the vicinity of the interface. We present a three dimensional calculation of the heat flux, and of the temperature distribution of the phonons in the solid, involving the solutions of the Boltzmann equation. We consider a metal in contact with liquid helium and essentially study the qualitative indirect (through phonons) contribution of the electrons to the Kapitza resistance. In a later paper, it is intended to show 
quantitatively the result of this approach on the Kapitza resistance.

2. Model calculation and approximations involved. - We will not report here the detailed equations $\left({ }^{1}\right)$ and calculations but only the essential features of the model. The present work uses the Boltzmann transport equations for phonons and electrons, in the steady state, with appropriate boundary conditions as starting points. This method allows the introduction of phonon lifetime effects. We only consider here the scattering of phonons by electrons : this important mechanism provides a frequency-dependent phonon mean free path. The phonon Boltzmann equation, with boundary conditions determined by the classical theory of elasticity, is solved without linearization, nor assumption about the form of the non-local occupation number $N(\mathbf{r}, \mathbf{q})$, and particularly about the existence of a characteristic, position (r) dependent, phonon temperature. These essential features, along with angular dependent conditions for the phonon transmission, yield a model which is quite different from some analytic attempts for solving the transport equation under particular conditions [17-20]. However, the electrons are assumed to be characterized by a local temperature $T_{\mathrm{e}}(\mathbf{r})$, calculated from the electron Boltzmann equation, energy exchange with phonons only being considered.

Since the medium considered is a metal, the transport of heat in the bulk is essentially due to the electrons. In this model, we do not allow for heat flux $J_{\mathrm{e}}$ by electrons across the solid-liquid helium interface $\left(J_{\mathrm{e}}(x=0)=0\right.$; Fig. 1). Therefore, phonons must transport all the heat at the interface. The coupled electron-phonon equations must be solved self-consistently so as to conserve the total heat flux $J$ every-

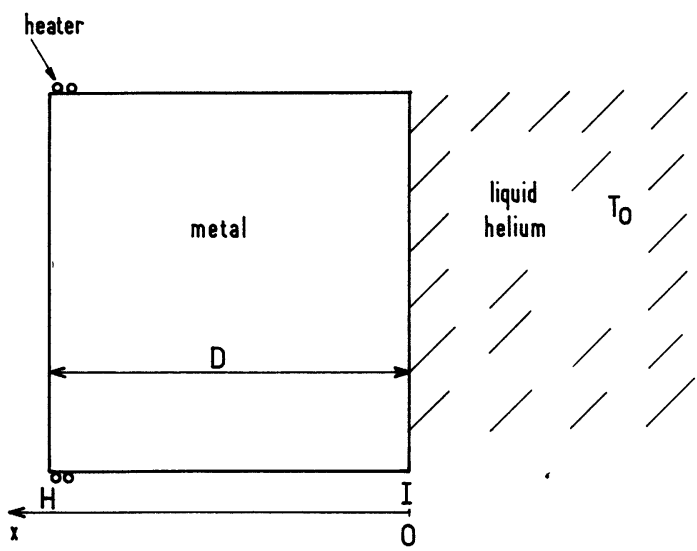

FIG. 1. - Configuration considered in the calculation of the phonon and electron heat fluxes and of the temperature distributions. $T_{0}=1 \mathrm{~K}$.

( $\left.{ }^{1}\right)$ The starting equations are essentially the same as the equations used in the spatial analysis of the electron-phonon system in a heater [16], except for the presence of an applied electric field. However, the two problems are quite different in the geometrical configuration and its resulting boundary conditions, in the ratio of the phonon mean free path to the specimen length... where in the metal in the steady state. In helium, we assume a constant temperature $T_{0}$.

3. Configuration model. - The calculation is carried out in a system (Fig. 1) as similar as possible to the usual experimental arrangement [6], i.e. a metallic slab (which we choose to be copper) in contact with liquid helium (interface I). An external heater provides a steady state common temperature for the excitations at the end $\mathrm{H}$ of the solid. The energy supplied by the heater flows, through the metal and the interface I, into the helium.

For the thickness of the slab $D$, let us adopt $500 \mu \mathrm{m}$. We assume the liquid helium temperature to be $T_{0}=1 \mathrm{~K}$.

4. Heat flux. - Considering only longitudinal phonons in the metal and using a Debye approximation, the phonon heat flux $J_{p}$ is given by the following equation :

$$
J_{\mathrm{p}}=\frac{\hbar u}{4 \pi^{2}} \int_{0}^{q_{\max }} \mathrm{d} q q^{3}\left[\int_{0}^{\pi / 2}\left(N_{\rightarrow}-N_{\leftarrow}\right) v_{x} \sin \theta \mathrm{d} \theta\right]
$$

$N_{\rightarrow}=N_{\rightarrow}\left(x, q, v_{x}\right)$ and $N_{\leftarrow}=N_{\leftarrow}\left(x, q, v_{x}\right)$ are the phonon densities at the point $x$, in the mode of wave vector $\mathbf{q}(q, \theta)$, moving respectively towards and from the interface $I$. In equation (1), $v_{x}=u|\cos \theta|$ is the projection of the phonon velocity on the $x$ direction $(\theta=(\mathbf{q}, \mathbf{O x}) ; 0 \leqslant \theta \leqslant \pi)$. When $N_{\rightarrow}$ and $N_{\leftarrow}$ are determined from the Boltzmann equation, the phonon heat flux is immediately known by numerical integration.

The electron heat flux $J_{\mathrm{e}}$ is given by :

$$
J_{\mathrm{e}}=-K_{\mathrm{e}} \cdot \nabla T_{\mathrm{e}}
$$

where $K_{\mathrm{e}}$ is the thermal conductivity. Using the Wiedemann-Franz law yields :

$$
J_{\mathrm{e}}=-\left(\pi^{2} k_{\mathrm{B}}^{2} \sigma /\left(3 e^{2}\right)\right) T_{\mathrm{e}} \frac{\partial T_{\mathrm{e}}}{\partial x}
$$

$k_{\mathrm{B}}$ is the Boltzmann constant and $e$ is the electron charge. The electrical conductivity $\sigma$ is in fact the residual conductivity $\sigma_{\mathrm{I}}$, the phonon contribution to the resistivity being very small and therefore neglected $[21,22]$. The thermoelectric force, which is necessary to ensure that the electrical current density is zero, is small and is neglected here.

The coupled equations for electrons and phonons are solved numerically. An electron temperature is assumed to be determined by the heater :

$$
T_{\mathrm{e}}(x=D)=T_{\mathrm{i}} .
$$

Then, an electron temperature gradient is chosen arbitrarily. The corresponding phonon densities are determined from the transport equation. The right values of $N_{\rightarrow}, N_{\leftarrow}$ and $T_{\mathrm{e}}$ are obtained by iteration, 
when their variations between two steps in the iteration become negligible. Moreover, these values must satisfy the equation of conservation of the total heat flux $J$,

$$
J=J_{\mathrm{p}}+J_{\mathrm{e}}=\text { const } .
$$

where $J_{\mathrm{e}}$ and $J_{\mathrm{p}}$ are calculated independently of each other, respectively by equations (2) and (1).

The main difficulty of the method lies in its convergence. It needs special care at the precision of each step in the calculation.

5. Results and discussions. - The essential features of the results are presented in figures 2 and 3 , for a helium temperature $T_{0}=1 \mathrm{~K}$ and a temperature $T_{\mathrm{i}}=1.0502 \mathrm{~K}$ imposed by the heater at the end $\mathrm{H}$ of the metal slab. The copper metal is assumed to be fairly pure $\left(\sigma=10^{10} \Omega^{-1} \mathrm{~m}^{-1}\right)$. The electron mean free path $l_{\mathrm{e}}$ is then about $10 \mu \mathrm{m}$. The total heat flux, $J=0.24 \mathrm{Wm}^{-2}$ (Fig. 2), resulting from these conditions, is constant within $10 \%$ over the whole length

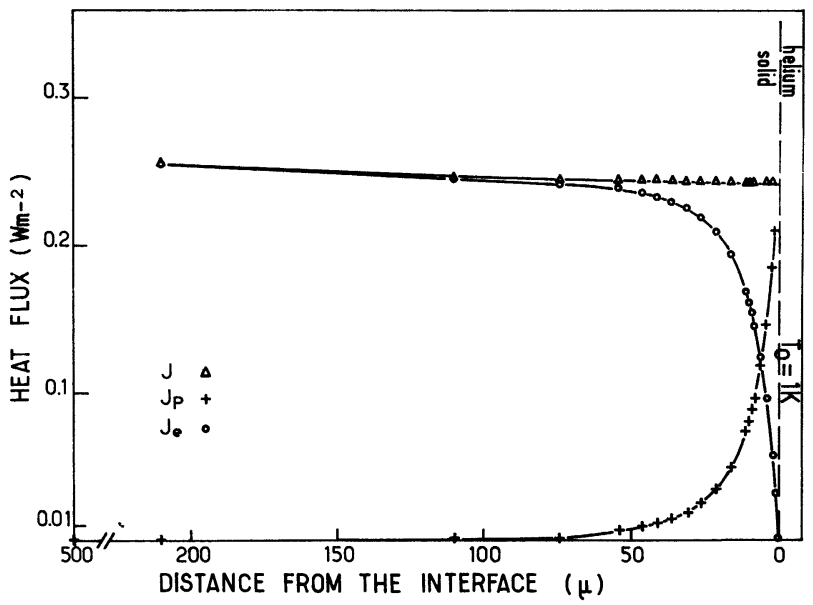

FIG. 2. - Heat fluxes of the electron $J(0)$ and of the phonons $J_{p}(+)$ as a function of the distance $x$ from the solid-liquid helium interface. The total heat flux $J=J_{\mathrm{e}}+J_{\mathrm{p}}$ is represented by the upper line. These curves are obtained after three iterations.

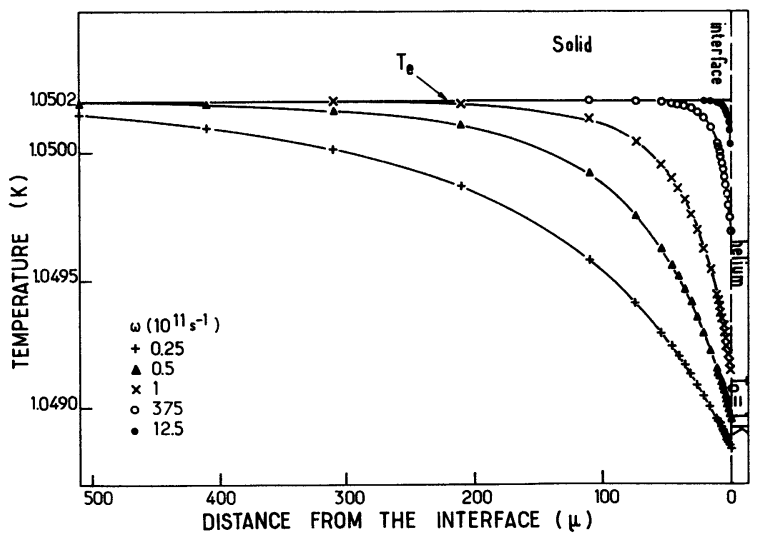

FIG. 3. - Angular averaged temperature $T_{\mathrm{p} \leftarrow}(x, \omega)$ of phonons for different frequencies $\omega$ moving a distance $x$ from the solidliquid helium interface. The upper curve represents the electron temperature. of the slab after three iterations. The better heat flux conservation ( $2 \%$ ) observed at a distance of about $100 \mu \mathrm{m}$ from the interface, is due to the more precise calculation in this region.

In figure 2 are also shown the electron and phonon heat fluxes, $J_{\mathrm{e}}$ and $J_{\mathrm{p}}$, as a function of the distance $x$ from the interface. As expected, a transfer of flux from the electrons to the phonons is observed when the interface is approached, the heat at the interface being transported by the phonons only. The distance over which this transfer occurs is about $100 \mu \mathrm{m}$, and is much larger than the electron mean free path $l_{\mathrm{e}}$. It is determined by the frequency dependent phonon mean free paths $l_{\mathrm{p}}$

$$
\begin{aligned}
& {\left[l_{\mathrm{p}}\left(\omega=0.25 \times 10^{11} \mathrm{~s}^{-1}\right) \simeq 225 \mu \mathrm{m}\right.} \\
& \left.\quad l_{\mathrm{p}}\left(\omega=12.5 \times 10^{11} \mathrm{~s}^{-1}\right)=4.5 \mu \mathrm{m}\right]
\end{aligned}
$$

and is of the order of the mean free path of the mode $q$ for which the flux density $\sum_{0 \leqslant \theta \leqslant \pi / 2} q^{3}\left(N_{\rightarrow}-N_{\leftarrow}\right)$ is maximum.

In figure 3 , the electron temperature is presented as a function of the position $x$ in the metal (upper line), along with phonon temperatures $T_{p \leftarrow}(x, \omega)$, characterizing the $\theta$ mean value of the occupation number of some modes of frequency $\omega=u q$, for phonons travelling from the interface $I$ towards the end $\mathrm{H}$ of the slab. These temperatures are defined by :

$$
\bar{N}\left(T_{\mathrm{p} \leftarrow}(x, \omega)\right)=\int_{0}^{\pi / 2} N_{\leftarrow} \sin \theta \mathrm{d} \theta
$$

where $\bar{N}(T)$ is the Planck distribution at the temperature $T$.

As expected from the large electron heat conductivity, the electron temperature gradient is nearly constant over the whole length of the slab and is about

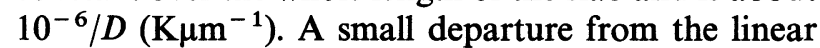
variation of the electron temperature with the distance $x$ is observed near the interface. The temperatures $T_{\mathrm{p} \rightarrow}(x, \omega)$ of the phonons moving towards the interface are not shown here : they are not significantly different from the electron temperature.

The phonon temperature $T_{\mathrm{p} \leftarrow}(x, \omega)$ is different from the electron temperature, particularly at the interface but also at a distance $x_{\omega}$ from the interface, which increases with decreasing phonon frequency. This can be understood by noting that the electronphonon relaxation time is frequency dependent $\tau_{\text {ep }} \propto \omega^{-1}$ [16]; therefore the energy exchange between the electrons and the phonons increases with the frequency. This results in a temperature $T_{\mathrm{p} \leftarrow}(x, \omega)$, monotonically increasing with frequency at a given $x$. The frequency variations of the temperature drop $\Delta T(\omega)=\left(T_{\mathrm{p} \leftarrow}(0, \omega)-T_{0}\right)$ observed at the interface are thus explained, the transmission 
coefficient depending on the direction of propagation, but not on the wave frequency in the acoustic mismatch model. Furthermore, the non-local character of the phonon transport explains the frequency variation of $x_{\omega}$, since the information received at the interface $\mathrm{I}$ by the emerging phonons is transported over distances of the order of a few phonon mean free paths $l_{\mathrm{p}}(\omega) \alpha \omega^{-1}$.

At a given frequency, the temperature $T_{\mathrm{p}}(x, \omega)$ shows non-linear variations with the position $x$ in the metal, at the distance $x_{\omega}$ from the interface. This clearly shows the influence of the phonon mean free path in the temperature distribution, and therefore in the Kapitza resistance. At distances $x>x_{\omega}$ from the interface $\mathrm{I}$, the phonon temperature $T_{\mathrm{p}}(x, \omega)$ reaches its bulk value.

According to the frequency dependence of $\Delta T(\omega)$, the contribution of the phonons to the Kapitza resistance depends on their frequency. The strong electronphonon coupling in the high frequency range yields large corresponding temperature drops $\Delta T(\omega)$, whereas small temperature drops are calculated in the low frequency range; the role of the electron-phonon interaction is then to increase the Kapitza resistance. This qualitative result is in agreement with experiments by L. J. Challis and M. C. Phillips on lead [15].

Experimentally, the temperature drop used in the determination of the Kapitza resistance is obtained by extrapolating the linear variation of the temperature in the bulk metal : it therefore corresponds to $T_{\mathrm{e}}(0)-T_{0}$ in our calculations, which is larger than the different drops $\Delta T(\omega)$. Therefore, a boundary resistance calculated from our $\Delta T(\omega)$, according to the Khalatnikov model, is expected to be a bit smaller than the measured one. However, our transport approach to the problem, which results in phonons being out of thermal equilibrium near the interface, needs the Kapitza resistance to be redefined, and does not allow here for an immediate quantitative comparison with the Khalatnikov theory. This will be presented with quantitative results elsewhere.
6. Conclusion. - The aim of our work was first to show that it is possible to obtain a numerical, rapidly converging, solution of the coupled transport equations for electrons and phonons; this solution satisfies both the boundary conditions and the condition of heat flux conservation.

Then, in a preliminary analysis, we have shown that the temperatures of the incident, $T_{\mathrm{p} \rightarrow}(x, \omega)$, and of the emergent, $T_{\mathrm{p} \leftarrow}(x, \omega)$, phonons at the interface I are quite different. Moreover, the variations of the gradients of the temperature $T_{\mathrm{p}_{\leftarrow}}(x, \omega)$ clearly show the importance of the phonon lifetime effects. The calculated phonon temperature gradients, which are seen to be non-constant over distances of the order of a few phonon mean free paths, must lead to a better agreement between the calculated Kapitza resistance and the experimental one. For a quantitative comparison with experiments, the Kapitza resistance must be redefined in the scope of this transport approach to the Kapitza problem, which is quite different from the Khalatnikov solution. However, we have shown here that the qualitative indirect contribution of the electrons to the Kapitza resistance is an increase of the Kapitza resistance, due to the electron-phonon interaction [15].

Information concerning the quantitative contribution of each mode $\mathbf{q}$ in the boundary resistance, as a function of its direction of propagation, as well as a function of its frequency, taking into account the density of states, can be deduced from these calculations. The influence of the boundary conditions can be studied. Still further, other mechanisms of phonon scattering could be included; the model could as well be applied to dielectrics with appropriate modifications.

Acknowledgments. - The author wishes to thank Professor L. Challis and Dr. F. Sheard of the University of Nottingham for helpful discussions during the course of this work. She also wishes to thank Professor J. Bok and Dr. J. Vannimenus for their useful comments on the results.

\section{References}

[1] Chaldis, L. J., J. Phys. C : Solid State Phys. 7 (1974) 481. [2] Anderson, A. C., Proceedings of the 2nd International Conference on Phonon Scattering in Solids, edited by Challis J. L., Rampton V. W. and Wyatt A. F. G. (Plenum Press) 1976 p. 1, and papers cited therein.

[3] Khalatnikov, I. M., Zh. Eksp. Teor. Fiz. 22 (1952) 687.

[4] Cheeke, J. D. N., J. Physique Colloq. 31 (1970) C 3-9.

[5] See $\S 4$ in [1] and references cited therein.

[6] Challis, L. J., Dransfeld, K. and Wilks, J., Proc. Roy. Soc. A 260 (1961) 31.

[7] Haug, H. and Weiss, K., Phys. Lett. A 40 (1972) 19.

[8] Peterson, R. E. and Anderson, A. C., J. Low Temp. Phys. 11 (1973) 639

[9] Opsal, J. L. and Pollack, G. L., Phys. Rev. A 9 (1974) 2227.

[10] Cheeke, D. and Ettinger, H., Phys. Rev. Lett. 37 (1976) 1625 .

[11] ANDrev, A. F., Sov. Phys. JETP 16 (1963) 1084.

[12] Little, W. A., Phys. Rev. 123 (1961) 435.

[13] SLUCKIN, T. J., preprint (1976).
[14] Wagner, F., Kollarits, F. J. and YaQub, M., Phys. Rev. Lett. 32 (1974) 1117.

[15] Challis, L. J. and Phillips, M. C., Proceedings of the 2nd International Conference on Phonon Scattering in Solids, edited by Challis L. J., Rampton V. W. and Wyatt A. F. G. (Plenum Press, New York) 1976, p. 28.

[16] Perrin, N., Solid State Commun. 17 (1975) 131.

[17] Budd, H. and Vannimenus, J., Phys. Rev. Lett. 26 (1971) 1637.

[18] Saslow, W. M., Phys. Rev. B 11 (1975) 2544.

[19] Phillips, M. C. and Sheard, F. W., Proceedings of the 2nd International Conference on Phonon Scattering in Solids, edited by Challis L. J., Rampton V. W. and Wyatt A. F. G. (Plenum Press) 1976, p. 24.

[20] Phillips, M. C., Ph. D. Thesis, Nottingham University (1974). Unpublished.

[21] Perrin, N. and Budd, H., Phys. Rev. Lett. 28 (1972) 1701.

[22] Perrin, N. and BudD, H., J. Physique Colloq. 33 (1972) C4-33. 